\section{Efetividade de diferentes estratégias de intervenção com ferro nos níveis de hemoglobina e ferritina em escolares de Teresina, Piauí, Brasil}

\author{
Effectiveness of different iron supplementation \\ strategies on hemoglobin and ferritin levels among \\ schoolchildren in Teresina, Piauí State, Brazil
}

\author{
${ }^{1}$ Departamento de Nutrição, \\ Universidade Federal do \\ Piauí, Teresina, Brasil. \\ 2 Universidade Federal de \\ Pernambuco, Recife, Brasil. \\ Correspondência \\ M. M. Santos \\ Departamento de \\ Nutrição, Universidade \\ Federal do Piauí. \\ Av. Senador Arêa Leão 2600 , \\ apto. 202, Teresina, PI \\ 64049-110, Brasil. \\ marizesantos@ufpi.br
}

\begin{abstract}
This study evaluated the effectiveness of supplementation with ferrous sulfate and iron bis-glycinate chelate on hemoglobin and serum ferritin levels among schoolchildren (7-11 years) of both sexes. A randomized community-based trial including 138 anemic children (hemoglobin $<11.5 \mathrm{~g} / \mathrm{dL}$ ) was conducted in Teresina, Piaui State, Brazil. Children were assigned to two treatment groups on an individual basis. One group $(n=71)$ received $40 \mathrm{mg}$ iron as ferrous sulfate once weekly and the other group $(n=67)$ received $3.8 \mathrm{mg}$ of iron bis-glycinate chelate-enriched cookies, 3x/week, for 8 weeks. The interventions showed a significant increase $(p<0.01)$ in hemoglobin levels $(1.1 \mathrm{~g} / \mathrm{dL})$ for children who received ferrous sulfate and $0.9 \mathrm{~g} / \mathrm{dl}$ in those who received iron bis-glycinate chelate, although not significant in the inter-group comparison ( $p>0.05)$. No effect was observed on body iron for either intervention ( $p>0.05)$. Children with depleted iron stores $(<15 \mathrm{ng} / \mathrm{mL})$ at the beginning of interventions showed increased serum ferritin concentrations after 8 weeks $(p<0.01)$, although no difference between treatments ( $p>0.05)$ was observed. The results confirm the effectiveness of the iron supplementation interventions and corroborate the use of iron salts or ferrous bisglycinate chelate on a weekly basis to overcome iron deficiency and anemia.
\end{abstract}

Iron; Anemia; Students
Marize Melo dos Santos 1

Nadir do Nascimento Nogueira 1

Alcides da Silva Diniz 2

\section{Introdução}

A anemia ferropriva representa, em nível mundial, o problema nutricional hegemônico em termos de saúde coletiva, afetando, principalmente, crianças e mulheres no ciclo reprodutivo 1. No Brasil, a anemia ferropriva tem assumido proporções epidêmicas, com tendência crescente nas últimas décadas 2,3. O Ministério da Saúde vem desenvolvendo estratégias para a redução da anemia ferropriva no país, a exemplo da suplementação medicamentosa e, sobretudo, da fortificação massiva das farinhas de trigo e milho, a partir de 20024.

A experimentação em animais tem demonstrado um aumento na biodisponibilidade do ferro, administrado em doses menores, diariamente, ou em doses intermitentes 5,6. Resultados similares vêm se confirmando na experimentação com humanos 7,8. Com relação à fortificação de alimentos, uma estratégia que vem sendo testada é o uso do composto quelato ${ }^{9}$, onde duas moléculas de aminoácido são utilizadas como ligantes, para impedir a ação de substâncias inibidoras. Nesse sentido, o ferro bisglicina quelato tem sido usado no enriquecimento de alimentos, com resultados promissores 10 .

Este estudo teve como objetivo comparar a efetividade entre a suplementação com sulfato ferroso e o consumo de biscoitos fortificados com ferro bisglicina quelato, em posologia semanal, nos níveis de hemoglobina e ferritina sérica em escolares. 


\section{Casuística e métodos}

Ensaio clínico comunitário, randomizado, para avaliar o efeito das suplementações com sulfato ferroso e com ferro bisglicina quelato nas concentrações de hemoglobina e ferritina sérica, envolvendo escolares de ambos os sexos, de 7 a 11 anos, matriculados em instituições públicas de ensino de Teresina, Piauí, Brasil, em agosto e setembro de 2000. Foram excluídos escolares que haviam feito uso de suplementos vitamínico e/ou mineral dois meses prévios ao início do experimento.

\section{Amostragem}

A amostra foi calculada baseada na variabilidade da distribuição dos níveis de hemoglobina (DP = $0,9 \mathrm{~g} / \mathrm{dL}$ ) 11 e em função do efeito esperado (hemoglobina $\geq 0,5 \mathrm{~g} / \mathrm{dL}$ ), adotando-se um erro $\alpha$ de $5 \%$ e um $\beta$ de $80 \%$ e utilizando-se a fórmula 12 : $\mathrm{N}=\left[\left(1 / \mathrm{q}_{1}+1 / \mathrm{q}_{2}\right) \mathrm{S}^{2}\left(\mathrm{Z}_{\alpha}+\mathrm{Z}_{\beta}\right)^{2}\right] / \mathrm{E}^{2}$. A amostra mínima necessária, para cada grupo, foi de 56 escolares. Para suprir perdas, corrigiu-se a amostra em $25 \%$. Utilizando-se uma tabela de números aleatórios, foram selecionadas 15 escolas, de um universo de 147, bem como a casualização dos 144 escolares elegíveis para o ensaio.

\section{Randomização}

Mediante processo de casualização simples, escolares com níveis de hemoglobina $<11,5 \mathrm{~g} / \mathrm{dL}$ foram alocados, individualmente, fazendo-se uso da tabela de números aleatórios, nos dois grupos de intervenção. Um grupo recebeu 40mg de sulfato ferroso, suspensão oral, dose única semanal, às 9 ou 14 horas, segundo o turno escolar (manhã/tarde); o outro grupo recebeu $3,8 \mathrm{mg}$ de ferro bisglicina quelato semanal, sendo a dose fracionada em seis biscoitos enriquecidos com ferro bisglicina quelato $(0,21 \mathrm{mg}$ /biscoito), perfazendo um aporte de 1,26mg de ferro bisglicina quelato/dia, consumidos no lanche matinal ou vespertino, três vezes na semana. O impacto das intervenções foi avaliado pelas concentrações das variáveis efeito (hemoglobina e ferritina sérica), transcorridas oito semanas de intervenção.

\section{Análises hematológica e bioquímica}

Mediante punção venosa cubital foram colhidos $6 \mathrm{~mL}$ ( $4 \mathrm{~mL}$ para análise da hemoglobina e $2 \mathrm{~mL}$ para ferritina sérica), após jejum noturno (12 horas). A coleta foi realizada na própria escola, sempre no horário matinal (8-10 horas), e as amostras enviadas ao Centro de Diagnóstico
Raul Bacelar, da Fundação Municipal de Saúde de Teresina para processamento e análise.

\section{Hemoglobina e ferritina sérica}

As concentrações de hemoglobina foram determinadas pelo método modificado de cianometohemoglobina e as concentrações de ferritina sérica pelo método de ensaio imunoenzimático 13 .

\section{Análise estatística}

Na comparação de proporções foram utilizados os testes do qui-quadrado (grupos independentes) e McNemar (amostras pareadas). Na comparação de médias foram empregados os testes $t$ de Student pareado (amostras dependentes) ou não-pareado (grupos independentes). Adotouse o nível de significância de $5 \%$ para rejeição da hipótese de nulidade.

\section{Aspectos éticos}

O protocolo de pesquisa foi aprovado pelo Comitê de Ética em Pesquisa, do Centro de Ciências da Saúde da Universidade Federal de Pernambuco (protocolo $\mathrm{n}^{\circ}$. 129/2000-CEP/CCS), atendendo às diretrizes e normas da Resolução $n^{o}$. 196/1996, para pesquisas envolvendo seres humanos. No rastreamento bioquímico foi encontrada uma prevalência de anemia superior à prevista no estudo, sendo, portanto, necessário o redimensionamento da amostra, o que levou à exclusão de algumas crianças para o seguimento do estudo de intervenção, que foram encaminhadas para tratamento ambulatorial. Ao final do estudo, os pais e/ou responsáveis foram informados sobre a situação de cada criança e aquelas que não conseguiram atingir concentrações adequadas de hemoglobina receberam sulfato ferroso ou biscoito com ferro bisglicina quelato, para darem continuidade ao tratamento em nível domiciliar.

\section{Resultados}

A comparabilidade entre os grupos de estudo mostrou distribuição homogênea em relação ao sexo $(p=0,36)$, concentrações de hemoglobina ( $p=0,52)$ e ferritina sérica $(p=0,65)$. Entretanto, observou-se que os escolares que receberam ferro bisglicina quelato apresentaram média de idade maior que aquela encontrada para o grupo que recebeu sulfato ferroso $(\mathrm{p}<0,01)$. As perdas, nos grupos sulfato ferroso $(\mathrm{n}=1)$ e ferro bisglicina quelato $(n=5)$, apresentaram as mesmas características dos escolares remanescentes no ensaio. A adesão ao tratamento foi extremamen- 
te elevada e o biscoito enriquecido teve excelente aceitação pelos escolares, uma vez que mais de 95\% das crianças consumiram integralmente os seis biscoitos oferecidos, nos três dias da semana, não sendo verificada rejeição por parte dos mesmos.

Ao final da intervenção, escolares suplementados com sulfato ferroso, bem como aqueles que receberam ferro bisglicina quelato tiveram um aumento significativo nas concentrações de hemoglobina (Tabela 1). Embora os dados apontem para uma tendência de maior incremento nas médias de hemoglobina, nos escolares tratados com sulfato ferroso esta diferença não foi significativa $(p=0,06)$. Entretanto, não foi observado nenhum impacto nas prevalências de hipoferritinemia $(<15 \mathrm{ng} / \mathrm{mL})$, tanto no grupo que recebeu sulfato ferroso (início $23,8 \%$ x final $18,8 \%$; $\mathrm{p}=0,63$ ) quanto no que recebeu ferro bisglicina quelato (início 23,9\% x final 14,9\%; $\mathrm{p}=0,11$ ).

Das 138 crianças que receberam sulfato ferroso ou ferro bisglicina quelato, $114(82,6 \%)$ conseguiram atingir concentrações satisfatórias de hemoglobina $(\geq 11,5 \mathrm{~g} / \mathrm{dL})$, ao final das oito semanas de intervenção. No entanto, a proporção de crianças tratadas com sulfato ferroso que apresentava hemoglobina $\geq 11,5 \mathrm{~g} / \mathrm{dL}$ foi similar $(\mathrm{p}=0,05)$ àquela de crianças que receberam biscoito com ferro bisglicina quelato. Por sua vez, as reservas inadequadas de ferro, ao final da intervenção, foram similares $(p=0,54)$ entre os escolares que receberam sulfato ferroso $(18,8 \%)$ e aqueles que receberam biscoito com ferro bisglicina quelato (14,9\%) (Tabela 2).

Dos 32 escolares que, no início do ensaio, apresentaram ferritina sérica $<15 \mathrm{ng} / \mathrm{mL}$, houve um incremento $(\mathrm{p}<0,01)$ nas médias de ferritina sérica, tanto no grupo que recebeu sulfato ferroso $(12,4 \mathrm{ng} / \mathrm{mL})$ quanto no grupo que recebeu biscoito com ferro bisglicina quelato $(5,6 \mathrm{ng} / \mathrm{mL})$. Embora os dados apontem para uma tendência de maior aumento nos níveis de ferritina sérica, nos escolares que receberam sulfato ferroso este incremento não foi significativo $(p=0,06)$ (Tabela 3).

\section{Discussão}

O impacto significativo na redução da anemia, tanto nos escolares que receberam sulfato ferroso quanto naqueles que receberam ferro bisglicina quelato, demonstra a efetividade dos tratamentos à base de ferro, seja em doses semanais (sulfato

Tabela 1

Concentrações de hemoglobina no início e após oito semanas de intervenção, em escolares de Teresina, Piauí, Brasil, 2000.

\begin{tabular}{|c|c|c|c|c|c|}
\hline \multirow[t]{2}{*}{ Grupos de intervenção } & \multirow[t]{2}{*}{$\mathrm{n}$} & \multicolumn{2}{|c|}{ Hemoglobina (g/dL) } & \multirow[t]{2}{*}{$p$ * } & \multirow[t]{2}{*}{ Incremento ( $g / d L)$} \\
\hline & & Inicial $(X \pm D P)$ & Final $(X \pm D P)$ & & \\
\hline Sulfato ferroso & 71 & $11,2 \pm 0,5$ & $12,3 \pm 0,8$ & $<0,01$ & $1,1 * \star$ \\
\hline Ferro bisglicina quelato & 67 & $11,1 \pm 0,7$ & $12,0 \pm 0,9$ & $<0,01$ & $0,9 * *$ \\
\hline
\end{tabular}

* Teste $t$ de Student (dados pareados);

${ }^{\star \star} p>0,05$; teste $t$ de Student (dados independentes).

Prevalência de anemia e reservas corporais inadequadas, após oito semanas de intervenção, em escolares de Teresina, Piauí, Brasil, 2000.

\begin{tabular}{|c|c|c|c|c|c|c|c|c|c|c|}
\hline \multirow[t]{3}{*}{ Grupos de intervenção } & \multirow[t]{3}{*}{$\mathbf{N}$} & \multicolumn{4}{|c|}{ Hemoglobina $<11,5 \mathrm{~g} / \mathrm{dL}$ * } & \multirow[t]{3}{*}{$\mathbf{N}$} & \multicolumn{4}{|c|}{ Feritina sérica $<15 \mathrm{ng} / \mathrm{mL}$ ** } \\
\hline & & \multicolumn{2}{|c|}{ Ausente } & \multicolumn{2}{|c|}{ Presente } & & \multicolumn{2}{|c|}{ Ausente } & \multicolumn{2}{|c|}{ Presente } \\
\hline & & $\mathbf{n}$ & $\%$ & $\mathrm{n}$ & $\%$ & & n & $\%$ & $\mathbf{n}$ & $\%$ \\
\hline Sulfato ferroso & 71 & 63 & 88,7 & 8 & 11,3 & 69 & 56 & 81,2 & 13 & 18,8 \\
\hline Ferro bisglicina quelato & 67 & 51 & 76,1 & 16 & 23,9 & 67 & 57 & 85,1 & 10 & 14,9 \\
\hline Total & 138 & 114 & 82,6 & 24 & 17,4 & 136 & 113 & 83,1 & 23 & 16,9 \\
\hline
\end{tabular}

$\chi^{2}: p=0,051$

${ }^{\star *} \chi^{2}: p=0,54$. 
Incremento nas concentrações de ferritina sérica, após oito semanas de intervenção, em escolares com depleção das reservas de ferro. Teresina, Piauí, Brasil, 2000

\begin{tabular}{|c|c|c|c|c|c|}
\hline \multirow[t]{2}{*}{ Grupos de intervenção } & \multirow[t]{2}{*}{$\mathrm{n}$} & \multicolumn{2}{|c|}{ Ferritina sérica $<15 \mathrm{ng} / \mathrm{mL}$} & \multirow[t]{2}{*}{$p$ * } & \multirow[t]{2}{*}{ Incremento (ng/mL) } \\
\hline & & Inicial (X $\pm \mathrm{DP})$ & Final $(X \pm D P)$ & & \\
\hline Sulfato ferroso & 16 & $7,3 \pm 4,8$ & $19,7 \pm 11,2$ & $<0,01$ & 12,4 ** \\
\hline Ferro bisglicina quelato & 16 & $10,3 \pm 2,7$ & $15,9 \pm 7,6$ & $<0,01$ & 5,6 ** \\
\hline
\end{tabular}

* Teste $t$ de Student pareado;

${ }^{\star \star} p=0,06$; teste $t$ de Student não-pareado.

ferroso), seja no esquema posológico de três vezes na semana (ferro bisglicina quelato). A similaridade na efetividade dos tratamentos, em termos de incremento nos níveis de hemoglobina, observada na nossa casuística, foi igualmente encontrada em crianças anêmicas da Guatemala 14 .

A literatura tem mostrado que doses de sulfato ferroso administradas semanalmente ou, pelo menos, duas vezes/semana, parecem apresentar melhor efetividade quando comparada à diária, em virtude do esquema posológico ser melhor tolerado pelos pacientes e, consequentemente, melhor adesão 7 . No entanto, em termos de eficácia, os resultados são ainda conflitantes 15 .

Na nossa casuística, o percentual de crianças anêmicas (16,9\%) que não respondeu ao tratamento com ferro, poder-se-ia atribuir ao fato de que o tempo de intervenção não teria sido suficiente para demonstrar um efeito terapêutico. No Estado de São Paulo, leite fluido fortificado com 3mg de ferro aminoácido quelato, ingerido diariamente, mostrou redução significativa na prevalência de anemia em crianças, após seis meses de intervenção ${ }^{15}$. No entanto, resultados similares foram observados com apenas dois meses de uso do ferro bisglicina quelato, adicionado à água do arroz e ao sachê de tempero do macarrão 16.

Por outro lado, a similaridade no impacto intertratamentos, nas concentrações de hemoglobina e ferritina sérica, poderia ser decorrente do fato de que a ingestão de ferro bisglicina quelato atingiu apenas $16 \%$ da recomendação diária, tomando-se como base um consumo de $8 \mathrm{mg} / \mathrm{dia} 17$, aportando, assim, uma quantidade de ferro significativamente menor do que a recomendada, além de ter sido administrado apenas três vezes/ semana.

A ausência de impacto das intervenções sobre os níveis de ferritina sérica poderia ser igualmente atribuída ao curto tempo de tratamento e/ou quantidade insuficiente de ferro consumi- da, fatores limitantes para a formação de reservas corporais adequadas. Nesse sentido, estudo conduzido por Giorgini et al. 10, onde pré-escolares que receberam pão fortificado com $2 \mathrm{mg}$ de ferro bisglicina quelato, duas vezes/dia, correspondendo a $40 \%$ das recomendações diárias, cinco dias/semana, durante seis meses, obtiveram aumento significativo $(8,9 \mu \mathrm{g} / \mathrm{L})$ nos níveis de ferritina sérica. Piñeda \& Ashmead 14 observaram também que apenas crianças suplementadas com ferro bisglicina quelato apresentavam incremento significativo nos níveis de ferritina sérica, atribuído à maior biodisponibilidade.

Vale salientar que o incremento significativo nos níveis de ferritina sérica, apenas nos escolares com reservas inadequadas (ferritina sérica $<15 \mathrm{ng} / \mathrm{mL}$ ), foi também demonstrado por Giorgini et al. 10 e seria um indício de que os esquemas de intervenção utilizados seriam apenas suficientes para recompor um quadro de deficiência de estoques, mas não o bastante para prover reservas corporais adequadas do mineral.

\section{Conclusão}

As intervenções com sulfato ferroso, uma vez/ semana, ou biscoito enriquecido com ferro bisglicina quelato, três vezes/semana, promoveram um aumento significativo nos níveis de hemoglobina, após oito semanas de suplementação. Esses resultados vêm confirmar a eficácia das intervenções com ferro no combate à deficiência do mineral e à anemia ferropriva; ratificar o uso do esquema de tratamento semanal; e recomendar a distribuição de sais de ferro e de veículos enriquecidos com ferro bisglicina quelato nas estratégias de prevenção e controle do estado carencial. 


\section{Resumo}

O estudo avaliou o efeito das intervenções com sulfato ferroso e com ferro bisglicina quelato nas concentrações de hemoglobina e ferritina sérica em escolares de 7-11 anos, de ambos os sexos, de Teresina, Piauí, Brasil. Foi desenvolvido ensaio clínico-comunitário, randomizado, envolvendo 138 escolares, com niveis de hemoglobina $<11,5 \mathrm{~g} / \mathrm{dL}$, alocados, individualmente, em dois grupos de tratamento. Um grupo $(n=71)$ recebeu $40 \mathrm{mg}$ de sulfato ferroso, uma vez/semana, e o outro ( $n=67) 3,8 m g$ de ferro bisglicina quelato, fracionados em biscoitos consumidos três vezes/semana, durante oito semanas. Houve um incremento $(p<0,01)$ médio, nas concentrações de hemoglobina, de 1,1g/dL entre os escolares que receberam sulfato ferroso e de $0,9 \mathrm{~g} / \mathrm{dL}$ para aqueles que receberam ferro bisglicina quelato, embora sem diferença $(p>0,05)$ na comparação intergrupos. Nenhum impacto foi observado $(p>0,05)$ nas reservas corporais de ferro. Entretanto, escolares que apresentaram depleção das reservas corporais de ferro $(<15 \mathrm{ng} / \mathrm{mL})$, no início dos tratamentos, tiveram aumento $(p<0,01)$ nas concentrações médias de ferritina sérica, após a intervenção, embora com efeito similar $(p>0,05)$ entre os grupos de tratamento. Os resultados confirmam a efetividade das intervenções e ratificam o uso do esquema semanal com sulfato ferroso e com ferro bisglicina quelato no tratamento da deficiência do mineral e da anemia ferropriva.

Ferro; Anemia; Estudantes

\section{Referências}

1. World Health Organization. Iron deficiency anemia: assessment, prevention, and control: a guide for program managers. Washington DC: World Health Organization; 2001.

2. Monteiro CA, Szarfac SC, Mondini L. Tendência secular da anemia na infância na Cidade de São Paulo (1984-1996). Rev Saúde Pública 2000; 34: 62-72.

3. Oliveira RS, Diniz AS, Benigna MJC, Miranda-Silva SM, Lola MMF, Gonçalves MC, et al. Magnitude, distribuição espacial e tendência da anemia em pré-escolares da Paraíba. Rev Saúde Pública 2002; $36: 26-32$.

\section{Colaboradores}

M. M. Santos foi responsável pela redação e revisão do artigo. A. S Diniz colaborou na redação final e revisão do artigo. N. N. Nogueira colaborou na revisão do artigo.

\section{Agradecimentos}

Os autores agradecem ao Laboratório Raul Bacelar da Fundação Municipal de Saúde de Teresina, pelo apoio técnico (exames laboratoriais) e medicamentos (sulfato ferroso); ao Grupo Mabel pelos biscoitos enriquecidos com ferro bisglicina quelato; e à Coordenação de Aperfeiçoamento de Pessoal de Nível Superior (CAPES), pelo fomento (bolsa de doutorado).
4. Agência Nacional de Vigilância Sanitária. RDC 344/2002. http://www.anvisa.gov.br/legis/resol/ 2002/34402rdc.htm (acessado em 05/Mai/2006).

5. Viteri FE, Liu X, Tolomei K, Martin A. The absorption and retention of supplemental iron is more efficient when iron is administered every three days rather than daily to iron normal and deficient rats. J Nutr 1995; 125:82-91.

6. Wright AJA, Southon S. The effectiveness of various iron-supplementation regimes in improving the Fe status of anemic rats. Br J Nutr 1990; 63: 579-85. 
7. Ferreira MLM, Ferreira LOC, Silva AA, Batista-Filho M. Efetividade da aplicação do sulfato ferroso em doses semanais no Programa Saúde da Família em Caruaru, Pernambuco, Brasil. Cad Saúde Pública 2003; 19:375-81.

8. Schultink W, Gross R, Gliwitzki M, Karyadi D, Matulessi P. Effect of daily versus twice weekly iron supplementation in Indonesian preschool children with low iron status. Am J Clin Nutr 1995; 61:111-5.

9. Ashmead HD. The chemistry of ferrous bisglycinate chelate. Arch Latinoam Nutr 2001; 51 Suppl 1:13-21.

10. Giorgini E, Fisberg M, De Paula RAC, Ferreira AMA, Valle J, Braga JAP. The use of sweet rolls fortified with iron bis-glycinate chelate in the prevention of iron deficiency anemia in preschool children. Arch Latinoam Nutr 2001; 51 Suppl 1:48-53.

11. Santos MM. Eficácia das intervenções com sulfato ferroso e com ferro bisglicina quelato no tratamento da anemia em escolares do Município de Teresina/PI, 2003 [Tese de Doutorado]. Recife: Universidade Federal de Pernambuco; 2003.
12. Hulley SB. Designing clinical research: an epidemiological approach. Baltimore: Willians \& Wilkins; 2001.

13. De Maeyer EM, Dallman P, Gurney JM, Hallberg L, Sood SK, Srikantia SG. Preventing and controlling iron deficiency anemia through primary health care: a guide for health administrators and program managers. Geneva: World Health Organization; 1989.

14. Piñeda OE, Ashmead HD. Effectiveness of treatment of iron-deficiency anemia in infant and young children with ferrous bis-glycinate chelate. Nutrition 2001; 17:381-4.

15. Torres MAA, Lobo NF, Sato K, Queiroz SS. Fortificação do leite fluido na prevenção e tratamento da anemia carencial ferropriva em crianças menores de 4 anos. Rev Saúde Pública 1996; 30:350-7.

16. Arraval SRM. Consumo de ferro suplementar no controle da anemia [Dissertação de Mestrado]. São Paulo: Faculdade de Saúde Pública, Universidade de São Paulo; 2001.

17. Batista-Filho M. Alimentação, nutrição e saúde. In: Rouquayrol MZ, Almeida NF, organizadores. Epidemiologia e saúde. 6a Ed. Rio de Janeiro: Medsi; 2003. p. 389-414.

Recebido em 04/Mai/2005

Versão final reapresentada em 29/Jan/2007

Aprovado em 14/Fev/2007 